\title{
Ce que les blogs d'enseignants disent de la lecture de bandes dessinées à l'école
}

\section{Hélène Raux}

\section{(2) OpenEdition \\ 1 Journals}

Édition électronique

URL : http://journals.openedition.org/trema/4826

DOI : $10.4000 /$ trema.4826

ISSN : 2107-0997

Éditeur

Faculté d'Éducation de l'université de Montpellier

\section{Édition imprimée}

ISBN : 979-10-96627-07-3

ISSN : 1167-315X

\section{Référence électronique}

Hélène Raux, "Ce que les blogs d'enseignants disent de la lecture de bandes dessinées à l'école », Tréma [En ligne], 51 | 2019, mis en ligne le 01 avril 2019, consulté le 06 août 2019. URL : http:// journals.openedition.org/trema/4826 ; DOI : 10.4000/trema.4826

Ce document a été généré automatiquement le 6 août 2019

Trema 


\title{
Ce que les blogs d'enseignants disent de la lecture de bandes dessinées à l'école
}

\author{
Hélène Raux
}

\section{Introduction}

1 Les documents d'accompagnement des programmes instituant explicitement en France l'enseignement de la littérature à l'école en 2002 indiquaient que la culture littéraire des élèves de l'école primaire devait se construire au fil d'un trajet varié et " permettre la rencontre des différents genres littéraires et éditoriaux habituellement adressés à l'enfance (albums, bandes dessinées, contes, poésie, romans et récits illustrés, théâtre) $»^{1}$ Depuis, les listes successives de lectures recommandées ${ }^{2}$ ont fait figurer un nombre croissant de titres de bandes dessinées parmi les lectures proposées. Pourtant, la place de ce médium semble rester très marginale dans les classes : des enquêtes par questionnaire font état d'une très faible représentation de bandes dessinées parmi les lectures d'œuvres intégrales à l'école (Louichon, 2008; Dardaillon, 2009; Bonnéry et al., 2015). Dans ce contexte de rareté, il n'est pas étonnant que les pratiques effectives relatives à la bande dessinée dans le cadre de l'enseignement de la littérature soient mal connues. Il importe pourtant de savoir comment les bandes dessinées sont exploitées quand elles franchissent la porte des classes, pour dépasser le constat d'une "mésentente » de la bande dessinée avec l'école (Berthou, 2015) et mieux comprendre ce qui se joue quand une bande dessinée est effectivement lue et travaillée en classe.

2 Pour approcher ces pratiques effectives, nous explorons un terrain peu fréquenté par les recherches en didactique : la blogosphère enseignante. Sur le plan méthodologique, les blogs d'enseignants offrent un accès facilité à des données en nombre qui permettent d'analyser une variété de cas en dépit de la rareté du médium dans les classes. Ils permettent en outre une position d'observation neutre qui ne modifie pas la teneur des données recueillies, et présentent parfois, autour des propositions d'exploitation 
pédagogique elles-mêmes, des commentaires et réflexions (de la part des blogueurs ou de lecteurs), qui apportent des éclairages complémentaires sur les ressources publiées. La nature des propositions qu'on y trouve doit cependant être questionnée : si elles semblent ancrées dans la pratique des enseignants qui les publient, il faut veiller à ne pas négliger les enjeux de communication et de représentation à l'œuvre dans un espace où se forme une «communauté de praticiens» (Henri et Pudelko, 2006) dans une dynamique de valorisation et d'expertise. A l'image des manuels fréquemment sollicités par les recherches en didactique, les blogs sont alors à envisager comme un «terrain de recherches» (Denizot, 2015), ce qui suppose une réflexion approfondie sur les caractéristiques de ce "terrain" et son rapport aux pratiques effectives. Avant de présenter les tendances observées en matière de bande dessinée dans les propositions consultées, l'article s'attachera donc à analyser les enjeux d'une investigation au sein de l'espace des blogs.

3 Parmi les très nombreux blogs consultables, l'échantillon retenu a été constitué par le réseau des blogs rattachés à la structure associative «Communauté des profs blogueurs ». Dans l'univers foisonnant, fragmenté et labile des blogs, cette communauté délimite en effet un périmètre à la fois large et susceptible d'une exploration exhaustive (elle regroupe quelque trois cents enseignants du premier degré dont tous ne traitent pas toutes les disciplines) et un ensemble de ressources diverses mais unifiées par une même démarche de partage de ressources variées entre pairs. Ce périmètre offre ainsi la possibilité d'observer le traitement d'un objet, ici la bande dessinée, au sein d'un « terrain » non spécialisé par rapport à cet objet, ce qui importe pour saisir des tendances à l'œuvre dans les pratiques ordinaires. Au final, les cent-vingt-trois blogs de ce réseau qui présentent des propositions en littérature pour les cycles 2 ou 3, pour lesquels des bandes dessinées sont recommandées dans les programmes, ont été passés en revue entre décembre 2016 et janvier 2017 : vingt-six séquences ont été recueillies, qui représentent sauf erreur de notre part l'ensemble des séquences spécifiquement consacrées à la bande dessinée dans cet échantillon.

Ce corpus d'enquête permet de traiter un ensemble de questions relatives à l'intégration de la bande dessinée à l'enseignement de la littérature à l'école élémentaire :

- Comment la bande dessinée est-elle lue dans les classes, avec quelles activités d'accompagnement? Dans quelle mesure ces activités s'inscrivent-elles dans des exercices habituels dans le cadre de la lecture littéraire ? Quelle place est accordée à l'image ?

- Quel corpus de bande dessinée est donné à lire aux élèves?

- Quelles représentations des enseignants s'expriment à travers les propositions d'exploitation pédagogique de bandes dessinées?

5 A travers les propositions recueillies, certes toujours singulières, l'analyse s'attachera à identifier si et comment elles "se calent sur de la régularité, de la typicité, de l'aspectualité et de la formalité » (Lahanier-Reuter, 2015), pour esquisser une «forme scolaire » de la bande dessinée, au sens de Denizot (2013) : les genres textuels et littéraires travaillés dans les classes de littérature sont des constructions disciplinaires, résultant d'un processus de scolarisation. Identifier la forme scolaire d'un genre littéraire, ou d'un médium, consiste selon ce cadre théorique à analyser comment il est reconfiguré à l'école, pour l'école, en examinant en particulier quelles œuvres sont les plus présentes dans les classes, quelle lecture en est faite et quels savoirs sont travaillés à l'occasion de cette lecture. 


\section{Les blogs d'enseignants, un terrain de recherches pour la didactique?}

6 Pour appréhender d'abord le statut des données recueillies sur des blogs, un point d'appui peut être trouvé dans les travaux consacrés aux manuels: ces travaux permettent de saisir ce que les blogs partagent avec cet autre support du curriculum proposé et ce qu'ils ont à offrir de spécifique au chercheur.

7 Si les manuels relèvent surtout de l'espace des "représentations disciplinaires " (Denizot, 2015), nous faisons l'hypothèse que les blogs se situent à la croisée de l'espace des représentations et de celui des pratiques ${ }^{3}$.

\section{Un contenu actualisé}

8 Alors que la consultation de manuels ne permet pas d'appréhender si, et comment, leurs propositions ont effectivement été actualisées dans les classes, les contenus présentés sur les blogs d'enseignants portent souvent la trace, au moins partielle, de leur actualisation. On trouve ainsi par exemple :

- des traces des activités menées, avec les documents utilisés, questionnaires de compréhension ou versions adaptées de l'œuvre, comme la blogueuse Maliluno, professeure des écoles, qui propose sur le Tour de Gaule, "pour les petits lecteurs, le texte des dialogues retapé comme une pièce de théâtre $»^{4}$; ce sont aussi des activités qui font suite à la lecture, comme la photo de coiffes indiennes réalisées par des CE2 à la suite de la lecture d'Anuki, bande dessinée sans texte dont le héros est un petit indien ${ }^{5} \ldots$

- des commentaires de l'enseignant lui-même, présentant une nouvelle version d'un document publié précédemment :

En période 2, nous allons travailler sur l'Antiquité. (cf. mon projet ici). Il y a quelques années, lorsque nous avions fait ce projet au travers du temps (voir ici), nous avions travaillé sur la BD d'Astérix: Le Tour de Gaule. Le souci c'est que nous l'avions travaillé en janvier et qu'entre un élève de CE1 de novembre et un de janvier, en lecture mine de rien il y a une grande différence... [...] Donc après de longues hésitations, $\mathrm{j}$ 'ai décidé de travailler cette $\mathrm{BD}$ quand même mais en retravaillant un peu le texte...6

- des bilans, proposés ou demandés dans les espaces de commentaires : Je voulais savoir si ton projet s'était bien passé?

- et oui ce projet s'est très bien passé ! le résultat est vraiment super, les élèves motivés et impressionnés par le rendu final. Ne pas hésiter à le faire ! ${ }^{7}$

Les propositions des blogs, on le voit à travers ces exemples, sont ancrées dans les pratiques des enseignants blogueurs et les blogs donnent partiellement à voir les conditions de leur actualisation ainsi que les réflexions qui les ont accompagnées.

\section{Des représentations exprimées explicitement}

10 Comme un manuel qui, situé dans l'espace des représentations disciplinaires, « révèle et véhicule des conceptions de l'enseignement et de la littérature » (Denizot, 2015, p. 44), un blog manifeste de telles conceptions. Mais sur les blogs, ces représentations disciplinaires ne se lisent pas qu'en creux des propositions pédagogiques, elles trouvent l'occasion de s'exprimer explicitement, en particulier dans les espaces d'échanges. 
11 On peut ainsi percevoir l'intérêt plus ou moins important suscité par une proposition, au travers du nombre de commentaires et de leur nature. Cet intérêt semble fonction du degré d'originalité du support de travail. Ainsi sur un même blog8, les séquences consacrées au Génie du Pousse-pousse de J.-C. Nogues et A. Romby ou à Otto, autobiographie d'un ours en peluche de Tomi Ungerer, albums très souvent travaillés par les enseignants, n'ont suscité que deux et trois commentaires (parfois espacés d'un an), quand une séquence sur l'adaptation en bande dessinée du Tour du monde en 80 jours ${ }^{9}$ en a suscité 29 en moins d'un an. Les visiteurs peuvent saluer le travail et remercier l'auteur, mais également demander des ressources complémentairescomme dans ces deux commentaires qui se suivent, quelques semaines après la publication de «fiches de lecture » pour le début de Popotka le Petit sioux sur le blog de lilipomme :

Merci encore pour ces précieux conseils et toutes ces fiches. Je vais commencer l'étude de Popotka avec mes élèves. Pensez-vous mettre la suite des fiches en ligne ou pas? A très bientôt !

Je suis suppléante dans une classe de CE1, j'ai beaucoup aimé les fiches sur la BD Popotka, je commence à travailler cet album avec mes élèves et j'aimerais savoir si je pourrais voir les autres fiches de l'album car je trouve qu'elles sont très bien faites. En vous remerciant par avance. ${ }^{10}$

12 L'intensité des échanges faisant suite à une proposition révèle des attentes plus ou moins fortes des enseignants selon les lectures et activités abordées : or les séquences observées recueillent de nombreux commentaires, signe que la bande dessinée figure parmi ces activités pour lesquelles un fort intérêt se manifeste.

De manière plus qualitative, s'expriment des représentations concernant les corpus d'œuvres enseignées, marquées en matière de bande dessinée par un certain « embarras pédagogique $»^{11}$ :

Merci beaucoup, j'ai la BD, mais je n'ai jamais osé l'exploiter en classe.

Cette année, j'ai des CM1 et ce sera plus facile avec vos fiches assez ouvertes! ${ }^{12}$

Super article! Merci... D'autant plus que je ne m’y connais pas tant que ça, alors tes

références sont précieuses ! ${ }^{13}$

Le fort intérêt qui s'exprime semble ainsi traduire non seulement un certain engouement pour la lecture de bandes dessinées, mais surtout les besoins des visiteurs des blogs dans ce domaine qui leur est peu familier et pour lequel ils sont particulièrement preneurs des rares ressources disponibles : le médium reste peu présent dans les classes, comme dans l'échantillon examiné, où la bande dessinée occupe une place proche de celle observée par les enquêtes précédemment citées, avec 26 séquences dans un ensemble de plus de 700 séquences de littérature. Le succès des propositions publiées est donc à la mesure de leur rareté et témoigne d'un fort besoin, de la part des visiteurs, d'outils sur lesquels s'appuyer pour travailler la bande dessinée.

15 Sur le plan méthodologique, la présence de ce dialogue entre les propositions des enseignants-blogueurs et les lecteurs qui les reçoivent renforce l'intérêt de cet espace d'échange professionnel pour appréhender la construction d'un objet scolaire : les blogs d'enseignants offrent un éclairage certain sur des aspects des pratiques effectives, qui dépasse les pratiques des seuls blogueurs. L'adossement des séquences publiées par des enseignants à leur pratique ne doit cependant pas faire oublier les enjeux de représentation inhérents à la situation de publication sur un blog. 


\section{Des pratiques ordinaires?}

16 De même que face aux ressources proposées dans des manuels, « la prise en compte de la spécificité du manuel comme système, ou comme objet interfaciel, paraît une nécessité » (Louichon, 2015, p. 25), il convient d'interroger la spécificité de la blogosphère comme système.

\section{Une « communauté de praticiens »}

L'écriture d'un blog professionnel est certes une initiative individuelle, mais elle s'inscrit dans la dynamique collective d'une "communauté de praticiens » définie par Henri et Pudelko (2006) comme le résultat de l' « engagement des individus dans la culture relié à une pratique professionnelle donnée » en vue « d'expliciter la pratique, de l'améliorer et même de la transformer » (p. 117). La motivation à participer à la "communauté de praticiens » peut être diverse :

désir de voir le domaine se développer, recherche d'interactions avec les pairs pour partager quelque chose d'important, désir de faire une contribution sachant qu'elle sera appréciée ou simple envie d'apprendre au sujet de la pratique dans une perspective personnelle de se perfectionner. (Henri et Pudelko, 2006, p. 117)

Ce sont bien les sources de motivation exprimées par les blogueurs et leurs lecteurs :

Professeur des écoles en CM1-CM2 depuis 2007, je pioche beaucoup de bonnes idées sur le net, à mon tour de partager $!^{14}$

Bonjour et merci pour ce rallye très intéressant, Je l'ai utilisé dans ma classe avec beaucoup de succès. Si ça t'intéresse, j'ai réalisé des fiches sur le modèle des tiennes pour d'autres BD ( Petit vampire va à l'école - Ludo, tranches de quartier - Samedi et Dimanche - Le génie de la boîte de Ravioli - Les trois chemins - Une sacré mamie). Je peux te les envoyer par mail. Au plaisir. Merci encore pour ces fabuleux travaux !15

19 La mise à disposition de ressources s'insère dans un réseau de partage : c'est bien une logique de réciprocité qui est à l'œuvre dans ces échanges, ce qui donne aux propositions inscrites dans ce réseau non pas une représentativité, impossible à évaluer, mais du moins une portée collective.

\section{Des enjeux de représentation}

20 La logique de réciprocité et la motivation à échanger n'exclut pas une autre logique, de l'ordre de la quête de reconnaissance ou de gratification.

Les membres qui donnent davantage deviennent de véritables personnalités de leurs petits mondes numériques. Ce qui permet de définir le deuxième facteur de participation: le degré de corrélation entre l'implication des membres d'une communauté en ligne et leur désir de reconnaissance publique. Si cette corrélation est forte, la communauté sera florissante. La soif de prestige pousse les usagers à continuer à donner de leur temps et à partager leurs contenus, d'une manière incrémentale, dans un cercle vertueux de reconnaissance et d'engagement. (Casilli, 2010, p. 54)

21 De fait, l'espace dévolu aux commentaires prend le plus souvent la forme d'un concert d'éloges. Dans la soirée qui a suivi la mise en ligne d'un conséquent dossier sur la bande dessinée, les commentaires ne se sont pas fait attendre pour complimenter l'auteur présent pour répondre :

Glaieul, 22 avril 19:09 : quel boulot !!! je suis admirative !! 
Romy, 22 avril 19:26: Merci ! Je ne suis pas aussi productive que certains et certaines et mes doc ne sont pas aussi aboutis mais je fais de mon mieux!

VEFA35, 22 avril 19:28 : Pfiou ! sacré travail ! bravo Romy, je vais regarder ça de près même si avec les ateliers d'écriture une fois par semaine, je vais piocher seulement des éléments ! Merci pour toutes ces recherches !!!

Romy, 22 avril 19:32 : (« smiley »)

Cato34, 22 avril 20:33: Fabuleux, quelle patience d'avoir réalisé toutes ces recherches! Une compilation et un travail impeccable ! Merci...

Romy, 22 avril 21:06 : Merci ces petits messages font vraiment plaisir !16 d'un investissement particulier. N'est-ce pas alors plutôt un espace d'expertise qui se développe, à distance des pratiques ordinaires? Au sein de la communauté de praticiens, on voit se jouer des positions privilégiées, s'affirmer des leaderships: l'entreréférencement, qui assure la reconnaissance entre membres, donne une plus grande visibilité aux blogs les plus suivis, ce qui produit un effet boule de neige. Les blogs les plus visibles peuvent alors devenir un tremplin pour des gratifications diverses: valorisation économique de nombreux blogs ayant mis en place un partenariat publicitaire avec la plateforme Amazon ${ }^{\circledast}$ (contre l'installation d'un lien commercial sur chaque couverture de livre présentée, l'auteur du blog touche une rémunération à chaque achat effectué par un internaute arrivé sur le site commercial depuis ce lien), ou publication de manuels à partir des ressources développées ${ }^{17}$. révèlent, et véhiculent, des manières d'enseigner la littérature; mais elles imposent de prendre en compte les dynamiques du système des blogs pour en interpréter les données. Les blogs sont alors à envisager comme un espace de mise en valeur d'une certaine idée des pratiques d'enseignement, plutôt que comme une fenêtre ouverte sur les pratiques du quotidien. La distance semble d'ailleurs si grande entre les propositions publiées et la réalité quotidienne qu'une campagne contre les effets pervers des ressources en ligne a vu le jour en 2016 au sein de la « communauté des profs blogueurs »:

Je suis enseignante, je suis potentiellement victime de burn-out, d'épuisement professionnel. [...] Parce que je vois sur internet des choses merveilleuses que je ne parviens pas à instaurer dans ma classe, nourrissant parfois ce sentiment d'incompétence. [.... ${ }^{18}$ Les blogueurs étaient alors invités à
écorner leur image d'enseignant parfait.

Les propositions pédagogiques disponibles sur les blogs peuvent donc se lire comme du contenu actualisé, mais faisant l'objet d'une valorisation : les séquences proposées n'en sont sans doute que plus révélatrices de la représentation que se font les blogueurs de ce qui mérite d'être publié, en vue d'être repris par la communauté. C'est donc moins comme un reflet de pratiques ordinaires que nous lirons les 26 séquences retenues sur un échantillon de la blogosphère, que comme un ensemble révélateur des représentations en vigueur concernant la façon dont la bande dessinée peut être lue en classe.

\section{Quelle(s) lecture(s) de la bande dessinée?}

Conformément à l'inscription de la bande dessinée comme un des "genres de textes » à lire dans les programmes de littérature, les séquences consacrées à la bande dessinée sont intégrées aux ressources relevant de la littérature. Pour la plupart (19), il s'agit d'un 
ensemble d'activités visant à accompagner la lecture intégrale d'un album. Indexées sous des onglets « littérature ", « lecture suivie » ou encore " lecture », ces séquences côtoient dans ces rubriques celles que l'enseignant-blogueur consacre à des romans ou à d'autres œuvres littéraires. Les sept autres séquences sont dédiées à « la bande dessinée » (titre le plus représenté, avec des variantes - « Zoom sur la BD »...). Si certaines sont placées dans des rubriques généralistes ("français») ou d'autres sous-domaines de la discipline (" maitrise de la langue » ou " production d'écrits»), elles s'inscrivent toutes, au moins partiellement, dans le cadre du travail de littérature. Par exemple, les objectifs figurant au début d'une séquence proposée en " maitrise de la langue $»^{19}$ sont en fait de :

- connaître la structure d'une bande dessinée

- savoir lire une bande dessinée

- écrire une bande dessinée d'après les dessins

- acquérir une culture de BD célèbres

Nous analysons donc la lecture mise en œuvre dans l'ensemble de ces séquences sous l'angle de la didactique de la littérature, en examinant en particulier comment la prise en compte de la spécificité du médium est articulée avec le travail de compréhension/ interprétation attendu dans l'enseignement de la littérature ${ }^{20}$.

\section{Lire une BD ou lire de la BD?}

Deux tendances s'observent principalement dans les séquences, qui peuvent paraître divergentes : d'un côté, une certaine banalisation de bandes dessinées parmi les lectures scolaires, donnant lieu à des questionnaires de compréhension similaires à ceux proposés sur des romans par exemple, et d'un autre côté une forte préoccupation de prise en charge des spécificités du genre, se traduisant par une étude systématique des codes de la bande dessinée souvent détachée de la lecture d'une bande dessinée particulière - dans les séquences portant sur la bande dessinée en général comme dans celles qui accompagnent la lecture d'un album. Dans cette oscillation entre invisibilité et hypervisibilité du médium ${ }^{21}$, nous lisons en fait deux manifestations d'une même difficulté à articuler prise en compte des caractéristiques du médium et travail sur le sens des œuvres: soit c'est une bande dessinée particulière qui est lue, mais l'attention se porte peu sur la narration visuelle; soit c'est la bande dessinée en général qu'il s'agit d'apprendre à lire au moyen d'une boîte à outils passe-partout.

Les questionnaires de compréhension (proposés dans 18 des 19 séquences de lecture d'album) n'accordent guère d'importance à l'appartenance des œuvres lues au genre bande dessinée. Ils s'attachent en effet peu à l'image et à la construction visuelle de l'histoire : les élèves sont invités à reformuler les enjeux de l'histoire ou les motivations des personnages et leurs relations, à identifier les étapes et la construction de l'intrigue en reconstituant un schéma narratif ou un schéma actanciel, mais rares sont les questions qui amènent à interpréter l'image. Ainsi, une carte d'identité des personnages du Sceptre $d^{\prime} O t t o k a r^{22}$ est à remplir, d'abord avec leur aspect physique puis leur caractère et leur comportement ; or, pour justifier leur choix pour ces derniers éléments, les élèves sont invités à "recopier des phrases du livre", comme si les éléments visuels n'étaient significatifs que de l'aspect physique des personnages, les seuls éléments à prendre en compte pour le caractère et le comportement étant textuels ${ }^{23}$. A l'instar de cet exemple, l'image est le plus souvent secondaire; le constat établi par Leclaire-Halté (2008) 
concernant le traitement des albums dans les instructions officielles de 2007 peut être repris :

C'est souvent le texte qui est mis en avant, l'image est au service de celui-ci, elle sert par exemple à rendre plus concrets aux yeux des jeunes lecteurs certains procédés littéraires (métaphore, ironie par exemple). Ou alors, elle contribue à la formation picturale des élèves (par une intericonicité avec des tableaux de peintres connus par exemple). En tout cas, elle est souvent présentée comme secondaire par rapport au texte. (p. 230)

Cette minoration de l'image est particulièrement forte quand les élèves sont amenés à lire l'album via un tapuscrit, ou à le transcrire en un texte rendant les images superflues : une consigne donnée sur John Chatterton détective ${ }^{24}$ est ainsi d' « écrire une phrase sous chaque image pour décrire ce qui se passe dans l'illustration. Le but est que quelqu'un qui ne connait pas l'album comprenne l'histoire en lisant votre script ». L'enseignante détaille le déroulement de l'activité :

Afin de donner plus de sens au travail, j'ai proposé aux élèves d'aller présenter et lire leurs scripts aux autres classes de l'école. En effet, si les classes comprennent l'histoire de John Chatterton à la lecture de leurs scripts c'est que l'objectif sera atteint. ${ }^{25}$

Il arrive tout de même que des questions appellent une lecture attentive de l'image et visent à expliciter le sens dont elle est porteuse. Elles concernent notamment des repères spatio-temporels ou des caractéristiques de l'énonciation : "pourquoi les dessins des pages 24 et 25 sont différents du reste de l'album?», ces pages représentant le rêve du héros ${ }^{26}$; «qui peut voir Pierre de cette façon, pourquoi? ${ }^{27} \ldots$ L'observation de conventions de la bande dessinée (comme les idéogrammes et lignes de mouvement) sert parfois la construction du sens: " on sait que les Indiens vont arriver avant de les voir arriver, pourquoi ? $\aleph^{28}$. Mais de telles questions attentives à l'image sont peu nombreuses (nous en avons relevé dans 6 des 18 questionnaires de compréhension étudiés) : dans l'ensemble, les aspects visuels de la narration sont largement minorés, l'étude de la spécificité du médium donnant souvent lieu à un autre type de travail, décroché, sur les codes de la BD.

15 séquences sur les 26 recueillies comportent ainsi un travail sur les composantes de la bande dessinée, sous la forme d'une fiche de vocabulaire. Une liste de termes est fournie, comprenant systématiquement le trio planche, vignette ou case, bulle, le plus souvent onomatopée et/ou cartouche, parfois idéogramme. Ces termes sont éventuellement accompagnés de définitions et de légendes, et les élèves doivent retrouver ces éléments comme dans cet exercice portant sur une planche des Petits mythos: « Entoure en rouge la planche, en vert une bulle, en bleu une vignette et en jaune le cartouche $»^{29}$. Dans cette approche de la spécificité du médium, ce sont des connaissances déclaratives qui sont visées, ces connaissances n'étant guère mise en relation avec la production du sens. Lors d'une évaluation en "lecture compréhension", les élèves sont ainsi évalués sur l'identification des composantes d'une vignette d'Astérix, sans avoir à en interpréter le sens $^{30}$. L'image (extraite du Tour de Gaule d'Astérix) représente la façade d'une boutique dans laquelle une intense bagarre a éclaté ; alors que cette bagarre n'est signifiée dans cette vignette que par le jaillissement d'onomatopées et d'idéogrammes qui semblent s'échapper par la porte et la fenêtre, les élèves sont invités à «colorier en rouge les onomatopées et en bleu les idéogrammes »: la signification de la vignette et l'effet produit par le choix d'une représentation indirecte de la bagarre ne sont nullement questionnés. 

jamais leur aspect visuel : l'exercice le plus courant (proposé dans 5 séquences) consiste à faire correspondre les onomatopées avec le bruit qu'elles transcrivent; une seule séquence les envisage comme de l'image, en proposant (en arts visuels) un collage d'onomatopées variées. En matière de spécificité du médium bande dessinée, la connaissance du vocabulaire spécifique et le décodage du texte pris isolément semblent donc primer sur l'appréhension du sens produit par la combinaison d'éléments textuels et visuels.

Au final, la narration visuelle n'est guère prise en compte par les deux modalités de travail les plus fréquemment rencontrées, qui apparaissent comme les deux facettes d'une même difficulté à mettre en place le travail de lecture et d'interprétation prévu par les programmes avec des textes comme avec des images ${ }^{31}$. Néanmoins, entre ces deux pôles, d'autres activités présentent également une certaine régularité: peut-être les mécanismes producteurs de sens de la bande dessinée y sont-ils davantage pris en compte?

Figure 1 : Occurrence de quelques activités dans les 26 séquences étudiées

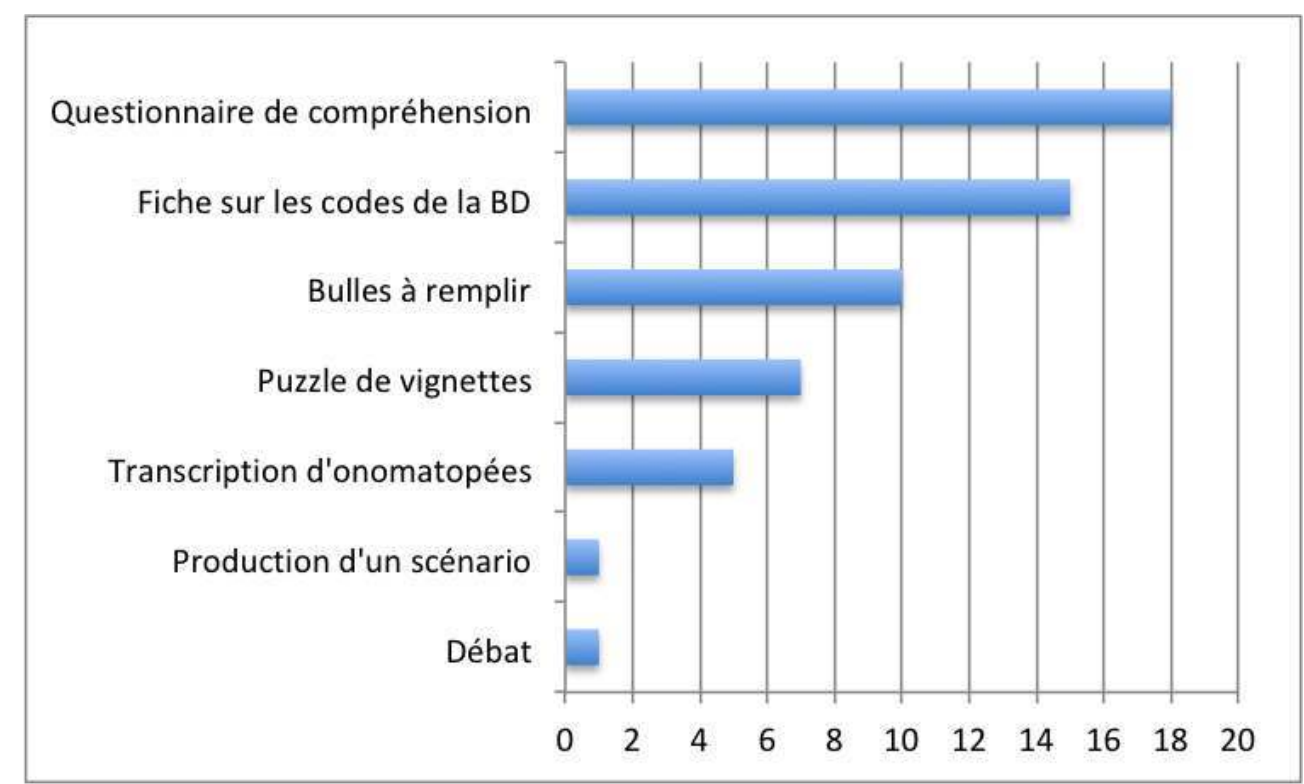

\section{Des exercices types pour comprendre et interpréter la bande dessinée?}

En dehors des questionnaires de compréhension et du vocabulaire de la bande dessinée, deux exercices-types apparaissent dans les séquences (cf Figure 2): le remplissage de bulles dont le texte a été effacé (relevé dans 10 séquences), et la mise en ordre des vignettes d'une planche à la façon d'un puzzle (dans 7 séquences).

Ces exercices ont été initialement promus lorsque la bande dessinée a commencé à être considérée comme digne d'un travail scolaire, notamment dans l'ouvrage pionnier $L a$ bande dessinée est éducative (Roux, 1970) :

L'entraînement à la déduction est facile à susciter par la fréquentation de la bande dessinée. Cet entrainement peut d'abord être provoqué par la création d'un 'puzzle' iconographique. [...] En classe, ce peut être le point de départ de discussions utiles 
entre des élèves qui n'auraient pas abouti au même résultat, et une amélioration de l'esprit d'induction et de déduction en leur faisant confronter leurs interprétations. [...]

A cheval sur la pratique d'une précision du vocabulaire et de l'entrainement à l'esprit de déduction serait l'emploi de bandes dessinées dont on aurait pris soin de "vider» les bulles de leur contenu : aux élèves d'imaginer les dialogues possibles. (p. 83)

Ils se sont par la suite largement imposés dans les ouvrages consacrés à l'exploitation de bandes dessinées, on les retrouve par exemple dans un guide pédagogique publié par les éditions ACCES (Schneider, 1998) auquel plusieurs blogueurs font référence, et ils se sont manifestement ancrés dans les pratiques des enseignants.

Le travail interprétatif des élèves, encouragé par la recherche comme par les programmes pour la lecture de littérature en classe, est-il mobilisé par ces exercices ? L'idée de débat est certes présente chez Roux, mais quelle place la reconstitution de la planche laisse-telle pour la discussion lorsqu'il s'agit de remettre les vignettes « dans l'ordre $»^{32}$, l'article défini excluant que plusieurs solutions puissent être proposées? Portant le plus souvent sur des planches humoristiques à chute, l'exercice est certes l'occasion d'une observation attentive des composantes de la planche pour repérer les indices aussi bien textuels que visuels de l'enchainement des vignettes, mais l'objectif de reconstitution de l'ordre initial ne peut engager de véritable débat autour de choix divergents.

Concernant la production écrite consistant à remplir des bulles, différentes modalités de l'exercice sont à distinguer. Imaginer les paroles des personnages peut participer d'un travail de compréhension/interprétation comme dans cette activité dans laquelle des bulles sont ajoutées par l'enseignante à des vignettes sans texte représentant les visages expressifs des personnages en gros plan, la consigne étant: «Imagine ce que pensent

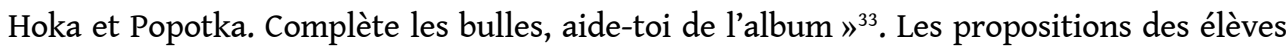
doivent ici s'appuyer sur une lecture attentive du passage, susceptible d'engager un phénomène d'identification aux personnages à partir duquel diverses pistes pourront être mises en discussion. Mais l'exercice est parfois abordé d'une toute autre manière, quand il s'agit de retrouver le texte qui a été gommé, au point que la confrontation des propositions des élèves avec la planche support soit qualifiée de " correction " $^{34}$. La même logique que celle du puzzle s'applique alors, qui vise très peu l'ouverture interprétative.

Ainsi, les modalités de travail qui s'affirment par ailleurs dans le domaine de la littérature, comme le débat interprétatif (Dias-Chiaruttini, 2008), semblent plus timidement mises en œuvre avec des bandes dessinées : une seule séquence, celle qui porte sur le Sceptre d'Ottokar, prévoit des moments de " débat », par exemple autour du passage où Tintin s'interroge sur la présence de la phrase «qui s'occupe des affaires d'autrui, s'expose à de graves ennuis » sur l'addition qu'on lui remet dans un restaurant au début de son enquête ${ }^{35}$. Les exercices éprouvés sur la bande dessinée, préconisés dès les années 1970 par les promoteurs de l'intégration de la bande dessinée aux supports de lecture scolaire, mobilisent certes des compétences de lecture du médium, mais ils s'intègrent rarement à une démarche de lecture interprétative. Dans l'ensemble, la particularité de la narration visuelle peine manifestement à être prise en charge dans un travail d'interprétation, négligé au profit d'approches très formelles ou d'un travail de compréhension littérale.

41 A l'image de certaines séquences visant à apprendre à lire «la BD », nous avons traité jusqu'ici le genre globalement. Ce n'est pourtant pas la bande dessinée qui est lue dans les 
classes, mais certaines bandes dessinées particulières, dont nous allons maintenant interroger le choix.

\section{Quelle bande dessinée?}

\section{Corpus des bandes dessinées sélectionnées par les blogs}

L'analyse se focalise ici sur les 19 séquences du corpus consacrées à la lecture d'un album intégral, car le choix d'une œuvre travaillée à l'échelle d'une séquence a toutes les chances d'engager des logiques plus profondes que le choix d'extraits traités très ponctuellement en vue d'objectifs plus restreints. Sont prises en compte, en complément, quelques mentions explicites d'une lecture suivie sur les 123 blogs consultés, pour lesquelles les activités mises en œuvre ne sont pas présentées (et n'ont donc pas pu être prises en compte dans l'analyse des modalités de lecture), ce qui explique un léger décalage entre le total des occurrences citées et le corpus précédemment décrit.

La première observation qu'on peut faire à propos des bandes dessinées retenues sur les blogs est que les choix suivent de manière contrastée les préconisations officielles : on trouve des titres reconduits par l'ensemble des listes de lectures recommandées publiées depuis 2002 en accompagnement des programmes de littérature, mais aussi, et majoritairement, des titres absents de ces listes, comme le montre le tableau ci-dessous qui répertorie les albums étudiés (présentés par ordre de fréquence, puis par ordre alphabétique du titre).

Tableau : Albums exploités en tant qu'œuvres intégrales

\begin{tabular}{|l|l|l|l|}
\hline Auteur & $\begin{array}{l}\text { Titre (année de } \\
\text { première } \\
\text { publication) }\end{array}$ & $\begin{array}{l}\text { Nombre } \\
\text { d'occurrences }\end{array}$ & $\begin{array}{l}\text { Recommandé par les listes } \\
\text { officielles }^{36} \text { ? }\end{array}$ \\
\hline Yvan Pommaux & $\begin{array}{l}\text { John Chatterton } \\
\text { Détective (1993) }\end{array}$ & 5 & $\begin{array}{l}\text { Mentionné comme prolongement } \\
\text { possible dans les notices de deux } \\
\text { romans policiers dans la liste de } \\
\text { 2004 pour le cycle 3. }\end{array}$ \\
\hline $\begin{array}{l}\text { David Chauvel, } \\
\text { Fred Simon }\end{array}$ & $\begin{array}{l}\text { Popotka le petit sioux } \\
\text { (2). Matho. (2004) }\end{array}$ & 2 & Non \\
\hline $\begin{array}{l}\text { Loïc Dauvillier, } \\
\text { Aude Soleihlac, } \\
\text { Anne-Claire } \\
\text { Jouvray }\end{array}$ & $\begin{array}{l}\text { Le Tour du monde en } \\
80 \text { jours (2013) }\end{array}$ & 2 & Non \\
\hline $\begin{array}{l}\text { René } \\
\text { Morris Goscinny, }\end{array}$ & $\begin{array}{l}\text { 7 histoires de Lucky } \\
\text { Luke (1991) }\end{array}$ & 1 & $\begin{array}{l}\text { Non } \\
\text { Frédéric Maupomé, La guerre des } \\
\text { Stéphane Sénégas } \\
\text { poules (2011) }\end{array}$ \\
\hline
\end{tabular}




\begin{tabular}{|c|c|c|c|}
\hline $\begin{array}{l}\text { René Goscinny, } \\
\text { Albert Uderzo }\end{array}$ & $\begin{array}{l}\text { Astérix le Gaulois } \\
(1961)\end{array}$ & 1 & Non \\
\hline Emile Bravo & $\begin{array}{l}\text { Boucle d'or et les sept } \\
\text { ours nains (2004) }\end{array}$ & 1 & Listes de 2007 et 2013 (cycle 2). \\
\hline $\begin{array}{l}\text { Roba } \\
\text { Verron, d'après } \\
\text { Roba }\end{array}$ & $\begin{array}{l}\text { Boule et Bill, Globe- } \\
\text { trotters (1982) } \\
\text { Boule et Bill, Mon } \\
\text { meilleur ami (2009) }\end{array}$ & 1 & $\begin{array}{l}\text { Non; le premier tome des gags de } \\
\text { Boule et Bill figure cependant } \\
\text { dans les listes du cycle } 2 \text { (2007 et } \\
\text { 2013). }\end{array}$ \\
\hline De Groot, Turk & $\begin{array}{l}\text { Léonard (29). Les bons } \\
\text { contes font les bons } \\
\text { génies. (1999) }\end{array}$ & 1 & Non \\
\hline $\begin{array}{l}\text { Christophe } \\
\text { Cazenove, Philippe } \\
\text { Larbier }\end{array}$ & $\begin{array}{l}\text { Les petits mythos (1) } \\
(2012)\end{array}$ & 1 & Non \\
\hline $\begin{array}{l}\text { Brigitte Luciani, } \\
\text { Eve Tharlet }\end{array}$ & $\begin{array}{l}\text { Monsieur Blaireau et } \\
\text { madame } \\
(2006)\end{array}$ & 1 & Listes de 2007 et 2013 (cycle 2). \\
\hline Mixelangelo Prado & Pierre et le Loup (1995) & 1 & $\begin{array}{l}\text { Listes de 2002, 2004, 2007, } 2013 \\
\text { (cycle 3). }\end{array}$ \\
\hline Hergé & $\begin{array}{l}\text { Le sceptre d'Ottokar } \\
(1939)\end{array}$ & 1 & Non \\
\hline Eric Omond, Yohan & $\begin{array}{l}\text { Toto l'ornithorynque et } \\
\text { l'arbre magique (1997) }\end{array}$ & 1 & $\begin{array}{l}\text { Listes de 2002, 2004, 2007, } 2013 \\
\text { (cycle 3). }\end{array}$ \\
\hline $\begin{array}{l}\text { René Goscinny, } \\
\text { Albert Uderzo }\end{array}$ & Le Tour de Gaule (1965) & 1 & Non \\
\hline
\end{tabular}
de dates de parution...), il semble que certaines logiques sous-tendant sa composition puissent être identifiées, notamment à la lumière du paratexte constitué par les commentaires des enseignants blogueurs, en présentation de leurs séquences ou dans leurs interventions sur les espaces d'échange.

\section{Séries du « panthéon » franco-belge}

Première tendance, les choix présentés reflètent d'abord les préférences partagées hors de l'école: Astérix, Tintin, Boule et Bill, Lucky Luke... c'est la bande dessinée la plus connue et la plus appréciée en France qui est proposée dans les classes - sondages ${ }^{37}$, expositions ou adaptations audiovisuelles témoignent régulièrement de l'attachement du public à ces bandes dessinées. 

propositions des recommandations officielles? On peut faire l'hypothèse que cela traduit l'absence d'habitudes scolaires liées au médium, les choix faits pour les classes ne s'écartant pas des représentations et des pratiques de lecture les plus partagées concernant la bande dessinée : on retrouve ainsi dans les classes les «bonnes vieilles BD de quand on était gamin, les Tintin, les Astérix, les machins comme ça " pour reprendre les propos d'un des interviewés de Lahire (2004, p. 247), qui synthétisent très bien les déclarations d'enquêtés de tous milieux et de toutes générations. motivations professionnelles, qui s'expriment parfois sur les blogs. Les grandes figures de la bande dessinée peuvent ainsi être convoquées pour leur intérêt culturel, leur appartenance au patrimoine :

[...] parler Antiquité sans parler d'Astérix en littérature, je trouve que c'est dommage.... les BD d'Astérix font partie de notre culture je trouve et pour le travail en DDM $^{38}$ sur la France, cette BD s'y prête super bien....39

Mais de telles justifications sont rares, et le plus souvent, c'est une représentation globale de la BD comme divertissement qui s'exprime, plutôt qu'une réflexion sur l'intérêt d'une bande dessinée particulière :

Mes élèves ont eu beaucoup de mal à écrire sur le premier trimestre alors j'ai voulu relancer leur motivation avec un projet un peu plus ludique ${ }^{40}$.

Le choix des œuvres est donc sans doute moins à analyser en partant des œuvres retenues qu'en fonction des préoccupations pédagogiques qui l'orientent. Or ces préoccupations semblent rejoindre celles qui président au choix de toute œuvre littéraire à exploiter en classe.

\section{Des bandes dessinées adaptées à l'organisation du travail en classe}

La forte représentation du registre humoristique qui apparaît dans la liste des titres présentée plus haut peut se lire comme le signe que les enseignants associent, conformément aux représentations dominantes, bande dessinée et humour ${ }^{41}$. Mais la nature des activités proposées pour accompagner la lecture en classe suggère que la logique du travail scolaire explique aussi l'intérêt des albums humoristiques : pour proposer une activité de puzzle avec des vignettes à remettre dans l'ordre, les planches à gags qui forment un ensemble d'une page indépendante se prêtent très bien à l'exercice... Les albums de Boule et Bill fournissent ainsi la majorité des supports pour l'exercice de puzzle dans les séquences consacrées à l'étude de la bande dessinée en tant que genre.

51 La même logique, conduisant à privilégier des supports se prêtant aisément à l'organisation des activités dans la classe, peut expliquer d'autres choix: une BD sans texte comme Anuki est ainsi appréciée car elle se prête très bien à un travail d'écriture - «ce sont des bandes dessinées sans texte (et donc bien sûr d'excellents supports de production d'écrit) $»^{42}$. Enfin, cette préoccupation peut être à l'origine de la forte représentation d'albums présentant des histoires de format court ou comportant des épisodes facilement identifiables : pour les 7 histoires de Lucky Luke, la lecture est accompagnée par des questionnaires de compréhension indépendants pour chaque histoire ou épisode ${ }^{43}$, c'est le cas également pour Popotka par exemple dont un récitatif marque nettement, toutes les deux ou trois pages, le découpage en épisodes ${ }^{44}$, ou pour le

Tréma, 51 | 2019 
Tour du monde en 80 jours dont chaque passage dans un pays ou une ville fait l'objet d'un questionnaire indépendant ${ }^{45}$.

Une autre considération liée aux contraintes de la classe s'exprime, la possibilité matérielle de mettre les bandes dessinées à la disposition des élèves :

Enfin une B.D. que l'on peut travailler sans mal en classe, et ce pour une raison tout

à fait matérielle : le prix. Ce tome (c'est le $2^{e}$ de la série) est édité dans la collection

Ribambelle CE1 et coute 3,50€ donc on peut facilement en équiper sa classe. On

retrouve le même genre d'ambiance que dans Yakari : de l'aventure, des légendes

indiennes, des sentiments... ${ }^{46}$

Dans la même perspective, le format atypique de John Chatterton pourrait également jouer un rôle dans son succès particulier : chaque page ne comporte qu'une ou deux vignettes de grand format, ce qui permet aux enseignants de mettre à disposition de tous les élèves l'intégralité de l'album en quelques photocopies réduisant la taille des pages ${ }^{47}$, la réduction des vignettes n'affectant pas leur lisibilité.

Un ensemble de préoccupations liées à la mise en œuvre pratique du travail dans la classe entre donc manifestement en jeu dans le choix des titres proposés aux élèves. D'autres préoccupations s'expriment sur le plan du contenu des œuvres et des possibilités d'exploitation offertes.

\section{Des bandes dessinées ouvrant sur une diversité d'exploitations}

Les deux titres les plus présents sur les blogs, John Chatterton et Popotka, illustrent deux orientations fréquentes de l'exploitation des albums : pour l'un une intertextualité riche, pour l'autre l'inscription dans un réseau thématique.

John Chatterton détective, réécriture du Petit Chaperon rouge dans un cadre emprunté au cinéma policier hollywoodien, se voit le plus souvent abordé pour une étude du genre policier, mais le fait qu'il s'agisse d'un conte détourné n'est pas négligé et cette double intertextualité ouvrant sur une diversité de rapprochements fait de l'album un support très intéressant :

Autres éléments d'analyse justifiant le choix de l'œuvre :

- L'ouvrage fait référence à d'autres œuvres littéraires (Le petit chaperon rouge p.13 ; Le petit poucet p.22).

- L'enquête (à lier avec le roman policier). ${ }^{48}$

C'est dans cette perspective d'intertextualité que des adaptations sont travaillées. Contrairement à ce qui a pu être observé à propos d'adaptations envisagées comme simples auxiliaires ou tremplins vers la lecture d'œuvres sources (Berthou, 2010), les adaptations choisies sur les blogs sont valorisées pour leurs qualités propres :

En parallèle avec l'écoute du conte musical de Prokofiev, mes élèves ont lu le superbe album de PRADO.

L'histoire de Pierre et le loup est illustrée de façon magnifique comme une bande dessinée.

La fin est une interprétation différente du conte qui met l'accent sur ce que ressent Pierre. ${ }^{49}$

De même, le choix du Tour du Monde en 80 jours est justifié par la qualité de la bande dessinée, «petit bijou graphique $»^{50}$. Ces deux séquences sur des adaptations prévoient des activités de confrontation avec l'œuvre source qui ne se réduisent pas à une simple comparaison pour établir points communs et différences: pour Pierre et le loup c'est 
l'interprétation qui est questionnée, et pour le Tour du Monde en 80 jours les élèves sont invités à observer le processus de l'adaptation en identifiant à partir d'un extrait du roman comment le scénariste a procédé pour découper la scène en vignettes. Les adaptations ne sont pas envisagées ici comme marchepied pour accéder au texte source, mais sont choisies pour leur capacité à entrer en dialogue avec l'œuvre adaptée, dans une dynamique où la lecture de l'une et de l'autre s'éclairent et s'enrichissent.

Une autre exploitation apparaît au cours de la lecture des albums ou dans leur prolongement: l'exploitation thématique avec souvent un volet documentaire. Popotka est ainsi intégré à un réseau sur les Amérindiens mis en relation avec un projet de découverte du monde ${ }^{51}$, Astérix mis à contribution pour aborder l'Antiquité, Toto l'ornithorynque pour "découvrir l'art des aborigènes d'Australie " ${ }^{52}$ - un commentaire renchérissant en termes d'exploitation documentaire, demandant si " $\mathrm{y}$ a moyen aussi d'en profiter pour bosser sur la classification animale ».

Que le choix de l'œuvre se fonde largement sur le thème abordé et les possibilités de mettre la lecture en relation avec des apprentissages dans une diversité de disciplines ou d'autres textes ne paraît pas spécifique aux œuvres de bande dessinée : les critères de choix que nous avons pu repérer rejoignent ceux identifiés par Dias-Chiarrutini (2008) concernant l'ensemble des textes littéraires, globalement «choisis en fonction de leur thématique, des disponibilités à l'école et par rapport aux activités et apprentissages qu'ils peuvent favoriser » (p.42), bien plus que pour la richesse du travail interprétatif engagé par l'œuvre. Sur ce plan, la bande dessinée apparaît logée à la même enseigne que l'ensemble de la littérature.

\section{Conclusion}

62 L'aperçu qu'offre la blogosphère enseignante sur les pratiques effectives de la littérature à l'école montre que des bandes dessinées sont lues et étudiées dans des classes. Moins que le nombre de propositions recueillies, assez faible au regard du nombre de blogs consultés, c'est l'intérêt manifeste suscité par ces propositions qui indique qu'un mouvement d'intégration de la bande dessinée aux œuvres intégrales est bien en cours : une forte demande de ressources s'exprime et les outils publiés suscitent un engouement certain. Concernant le corpus des bandes dessinées choisies, on observe d'ailleurs des logiques similaires à celles qui sont à l'œuvre dans l'enseignement de la littérature en général, avec la prédominance de critères thématiques ou l'importance de considérations liées à l'organisation pratique du travail de la classe. Du côté des activités accompagnant la lecture en classe, le médium peine toutefois à faire l'objet d'un travail de lecture littéraire plaçant les élèves en situation de questionnement : entre un travail très formel sur les codes du genre et des questionnaires de compréhension littérale accordant peu de place à l'image, on ne voit que rarement l'ensemble des composantes des œuvres prises en compte dans un questionnement ouvert engageant un travail interprétatif. Pour la recherche et la formation, le chantier de l'intégration d'œuvres multimodales au cadre de la lecture littéraire reste largement ouvert. 


\section{BIBLIOGRAPHIE}

Berthou, B. (2010). Adaptations : une certaine littérature. Du9, janvier 2010. https://

www.du9.org/dossier/adaptations-une-certaine/

Berthou, B. (2015). Bande dessinée, école et université : quelle mésentente ? Comicalités. https://

graphique.hypotheses.org/537

Bonnéry, S., Crinon, J., Marin, B. (2015). Des inégalités d'usage de la littérature de jeunesse dès les premiers cycles de l'école primaire? Une enquête par questionnaires. Spirale. Revue de recherches en éducation, $\mathrm{n}^{\circ} 55$. Supports et pratiques d'enseignement : quels risques d'inégalités ?, 43-56.

Casilli, A. (2010). Les liaisons numériques vers une nouvelle sociabilité ? (La couleur des idées). Paris: Éditions du Seuil.

Dardaillon, S. (2009) Quelle place pour l'iconotexte dans les pratiques des enseignants du cycle 3 ? La Littérature en corpus, Louichon, B. et Rouxel, A. Dijon : Scéren-CRDP Bourgogne, 85-95.

Denizot, N. (2006). Le biographique au lycée : vie et mort d'un genre scolaire. Recherches, 45, 187208.

Denizot, N. (2013). La scolarisation des genres littéraires 1802-2010. Bruxelles Bern Berlin: Peter Lang.

Denizot, N. (2016). Le manuel scolaire, un terrain de recherches en didactique ? L'exemple des corpus scolaires. Le français aujourd'hui, 194,(3), 35-46.

Dias-Chiarrutini, A. (2008). Que disent les enseignants du cycle 3 de leurs pratiques et des textes qui instituent la littérature à l'école primaire ? Pour une analyse des déclarations de pratiques.

Repères, 37, 27-49.

Groensteen, T. (1999). Le système de la bande dessinée. Paris : PUF.

Henri, F. et Pudelko, B. (2006) Le concept de communauté virtuelle dans une perspective d'apprentissage social. In Comprendre les communautés virtuelles d'enseignants. Pratiques et recherches. Daele, A. et Charlier, B. Paris : l'Harmattan.

Lahanier-Reuter, D. (2015) Contenus disciplinaires : questions d'échelles et d'espaces. In Les contenus d'enseignement et d'apprentissage approches didactiques. Daunay, B., Fluckiger, C., \& Hassan, R. (Etudes sur l'éducation). Pessac: Presses Universitaires de Bordeaux.

Lahire, B. (2004). La culture des individus. Dissonances culturelles et distinction de soi. Paris: La Découverte.

Leclaire-Halté, A. (2008). Place et traitement des genres fictionnels de littérature de jeunesse dans les programmes de l'école primaire de 2007. Pratiques, 137-138, 217-234.

Louichon, B. (2008). Enquête sur le rapport des enseignants aux programmes de 2002 et les pratiques d'enseignement de la littérature au cycle 3. Repères. Recherches en didactique $d u$ français langue maternelle, (37), 51-68.

Louichon, B. (2015) Essai d'analyse de l'analyse de manuels. In Perret-Truchot L. Analyser les manuels scolaires. Questions de méthodes. Rennes: PUR, 17-31. 
Martel, V. et Boutin, J.-F. (2015) La bande dessinée comme vecteur de coopération disciplinaire et éducationnelle. Biennale internationale de l'Education, de la Formation et des pratiques professionnelles. Paris, France. halshs-01172145

Reuter, Y. (2007). La conscience disciplinaire. Education et Didactique, 1-n², 55-71.

Rouvière, N. (2012). Etudier une oeuvre intégrale en bande dessinée au cycle 3 : quelles spécificités didactiques? In Bande dessinée et enseignement des humanités. Grenoble: Ellug, 103-121.

Roux, A. (1970). La bande dessinée peut être éducative. Paris: L'Ecole.

Schneider, J.-B. (1998). Clés pour la BD : Lire, analyser, produire avec les enfants de 8 à 15 ans. Accès Editions.

Siméon, J.-P. (1996) Lecture de la poésie à l'école primaire. Une démarche possible : la lecture d'une œuvre poétique complète. Repères, 13, 131-146.

\section{NOTES}

1. Littérature, cycle des approfondissements (cycle 3). Ministère de la Jeunesse, de l'Education nationale et de la Recherche. Direction de l'enseignement scolaire. CNDP, 2002, p. 5.

2. Listes publiées par le ministère de l'Education nationale en 2004, 2007, 2013 et 2015.

3. Selon les espaces disciplinaires définis par Reuter (2007), qui distingue espaces des prescriptions, des recommandations, des pratiques et des représentations.

4. Blog de Maliluno : http://maliluno.eklablog.com/litterature-le-tour-de-gaule-d-asterixa119083264, consulté le 3 janvier 2017.

5. Anuki, Stéphane Sénégas et Frédéric Maupomé, La Gouttière, 2011. http://www.caracolus.fr/ art-anuki-et-les-indiens/, consulté le 9 janvier 2017.

6. Blog de Maliluno précédemment cité. C'est nous qui soulignons les passages concernant les reprises de propositions antérieures.

7. Echange concernant un travail sur Popotka le petit Sioux, David Chauvel et Frédéric Simon, Delcourt, 2004 : http://www.lilipomme.net/popotka-le-petit-sioux-mahto-a28622815, Consulté le 10 janvier 2017.

8. L'école d'Alexandre : http://cancandre.eklablog.com/, Consulté le 4 janvier 2017

9. Loïc Dauvillier et Aude Soleilhac, Delcourt, 2008.

10. http://lilipomme.net/popotka-le-petit-sioux-mahto-a28222815. Consulté le 10 janvier 2017.

11. Notion proposée par Siméon (1996) à propos de la poésie devant laquelle les enseignants se disaient démunis.

12. Commentaire de galloune à la séquence sur Pierre et le Loup de Mixelangelo Prado publiée par Caracolus : http://www.caracolus.fr/pierre-et-le-loup-de-prado/. Consulté le 9 janvier 2017.

13. Commentaire au "Zoom sur la BD», http://mitsouko.eklablog.com/zoom-sur-la-b-da78380179 Consulté le 10 janvier 2017.

14. alicecyle3 auteur du blog http://mamaitressedecm1.fr/?author=1. Consulté le 12 décembre 2016.

15. eurielle sur http://lecoledepetiteprune.eklablog.com/rallye-bandes-dessinees-a4614752. Ces propositions ont ensuite été intégrées sur la page citée, identifiées comme "une généreuse contribution d'eurielle ! ». Consulté le 18 juin 2017.

16. http://www.lepetitcoindepartagederomy.fr/francais-du-dialogue-a-la-bande-dessineecycle-3-partie-2-la-bande-des-a84200288. Consulté le 13 décembre 2016. 
17. Par exemple, les éditions SEDRAP proposent «Filou mène l'enquête, un outil numérique par l'auteur du blog Le cartable de Séverine. Des missions ludiques qui favorisent la compréhension de différents types d'écrits ».

18. Par exemple http://val10.eklablog.com/epuisement-professionnel-tous-concernes-des-profsblogueurs-s-engagent-a125242844. Consulté le 22 juin 2017.

19. http://titicole.canalblog.com/archives/2009/02/19/12618188.html Consulté le 16 décembre 2016.

20. Les programmes de 2008 , en vigueur lors de la publication de la plupart des séquences recueillies, explicitent à quelles démarches d'interprétation la lecture de textes littéraires peut donner lieu, au-delà du décryptage d'un sens univoque. Par exemple : «les œuvres en débat: approche de l'interprétation des textes ", dans Une culture littéraire à l'école, MEN/DGESCO, mars 2008, pp. 5-6.

21. Denizot (2006) remarque que le biographique a été longtemps scolarisé mais de manière invisible, étant présent dans les manuels sans faire l'objet d'une étude spécifique en tant qu'extrait d'autobiographie ; nous reprenons ce terme et proposons le pôle inverse pour la bande dessinée quand celle-ci n'est au contraire abordée que par le prisme de ses codes spécifiques.

22. Hergé, Casterman, 1947.

23. La classe de Carole, http://ekladata.com/zBvqTUC4LatJli539os56PybSLM/Parcours-1.pdf Consulté le 15 décembre 2016.

24. Yvan Pommaux, Ecole des loisirs, 1993. L'ouvrage est en fait à la frontière entre l'album et la bande dessinée: chaque page présente ce qui s'apparente à une ou deux vignettes de bande dessinée en très grand format, les personnages s'exprimant dans des bulles et aucun autre texte n'accompagnant le récit. Les codes sont donc bien ceux de la bande dessinée, mais la séquentialité des images s'établit d'une page à l'autre plutôt qu'au sein du multicadre caractéristique du système de la bande dessinée (Groensteen, 1999). Malgré cette particularité, nous prenons pleinement John Chatterton en considération dans notre étude : non seulement l'attribution du prix littéraire allemand Max et Moritz de la «meilleure bande dessinée pour enfants » à cet album en 1996 marque sans ambiguïté son appartenance au champ de la bande dessinée, mais surtout la proximité de l'ouvrage avec l'album n'empêche en rien d'examiner comment ce qu'il comporte de bande dessinée est traité par les enseignants.

25. http://maisquefaitlamaitresse.com/john-chatterton/ Site consulté le 11 juillet 2018.

26. http://mamaitressedecm1.fr/?p=1884 Site consulté le 4 janvier 2017.

27. http://www.caracolus.fr/pierre-et-le-loup-de-prado/ Site consulté le 9 janvier 2017.

28. http://boutdegomme.fr/ekladata.com/boutdegomme.eklablog.com/perso/-CM-LECTURE/ Lecture-Suivie-7-Histoires-de-Lucky-Luke-b-lig.pdf Site consulté le 14 décembre 2016.

29. http://cenicienta.fr/la-mythologie-bd-les-petits-mythos-t1/ Site consulté le 14 décembre 2016.

30. Contribution de marie-amélie sur le blog de maliluno : http://ekladata.com/-JppLb65QtdJaduCfockNgI0jg/asterix-eval-tour-de-Gaule-contribution-marie-amelie.pdf Site consulté le 17 décembre 2017.

31. Les programmes de 2008 , en vigueur lors de la publication de la plupart des séquences consultées, envisagent la bande dessinée dans ce cadre : «Dans l'album ou la bande dessinée, [l'illustration] joue souvent un rôle encore plus décisif que le texte. C'est donc bien l'ensemble texte/images qui, le plus souvent, doit être compris et interprété. " Une culture littéraire à l'école, MEN/DGESCO, mars 2008, p. 4.

32. Les consignes varient quelque peu mais «l'ordre» y est systématiquement mentionné. Quelques exemples des variantes de la consigne : « Replace les vignettes dans l'ordre » sur http:// cenicienta.fr/wp-content/uploads/Telechargements/CM/Litterature/mytho-BD/Documents-

Litterature-Les-petits-mythos.pdf Consulté le 10 janvier 2017 ; «Colle les images dans l'ordre de 
l'histoire ", sur http://cycle2.orpheecole.com/2012/02/lecture-litterature-cp-et-ce1-monsieurblaireau-et-madame-renarde-p-4/\#lightbox[2832]/5/ Consulté le 14 décembre 2016.

33. Fiche de lecture 2 sur Popotka, le Petit Sioux, issue de http://www.lilipomme.net/popotka-lepetit-sioux-mahto-a28622815. Consulté le 10 janvier 2017.

34. Par exemple dans le projet « maitrise de la langue » de titicole : «Correction : lecture de la page réelle ». http://p7.storage.canalblog.com/78/89/420257/36105088.doc Consulté le 16 décembre 2016.

35. La classe de Carole, http://ekladata.com/zBvqTUC4LatJli539os56PybSLM/Parcours-1.pdf Consulté le 15 décembre 2016.

36. Les propositions consultées étant souvent datées de plusieurs années, nous avons pris en considération les différentes listes publiées entre 2002 et 2016, soit les listes de 2002, 2004, 2007 et 2013 pour le cycle 3 et de 2007 et 2013 pour le cycle 2 .

37. Astérix, Tintin, Lucky Luke, Boule et Bill et Gaston Lagaffe apparaissent comme les séries préférées des Français, dont $86 \%$ déclarent les « séries traditionnelles franco-belges 》 comme leur type de BD préféré. D'après un sondage BVA réalisé en janvier 2017. http://www.bva.fr/fr/ sondages/observatoire_de_la_vie_quotidienne_-_les_francais_et_la_bande_dessinee.html site consulté le 13 juillet 2018.

38. Découverte du monde, domaine qui regroupe les apprentissages visant notamment à se repérer dans le temps et l'espace.

39. http://maliluno.eklablog.com/litterature-le-tour-de-gaule-d-asterix-a119083264, consulté le 3 janvier 2017.

40. http://www.loustics.eu/lecture-expression-ecrite-la-bd-a104765074 Consulté le 16 décembre 2016.

41. " C'est le genre humoristique que préfèrent les Français (57\%), devant l'aventure (36\%) et la BD jeunesse (26\%). On ne note pas de différences ici entre sexes et générations, l'humour s'imposant partout. » Sondage BVA cité ci-dessus.

42. http://lutinbazar.fr/escale-en-amerique-du-nord/ Consulté le 14 décembre 2016.

43. http://boutdegomme.fr/ekladata.com/boutdegomme.eklablog.com/perso/-CM-LECTURE/ Lecture-Suivie-7-Histoires-de-Lucky-Luke-b-lig.pdf Consulté le 14 décembre 2016.

44. Par exemple http://le-stylo-de-vero.eklablog.com/les-livres-de-ribambelle-ce1-a107807604 Consulté le 13 juillet 2018.

45. http://cancandre.eklablog.com/tour-du-monde-en-80-jours-le-a125037930 Consulté le 16 décembre 2016.

46. http://lutinbazar.fr/escale-en-amerique-du-nord/ Consulté le 14 décembre 2016.

47. La classe de Kado propose par exemple une version de l'album en 3 pages noir et blanc. http://kalolanea.hautetfort.com/tag/john+chatterton

48. http://maliluno.eklablog.com/les-enquetes-policieres-a2284653 Consulté le 13 juillet 2016.

49. http://www.caracolus.fr/pierre-et-le-loup-de-prado/ Consulté le 9 janvier 2017.

50. http://cancandre.eklablog.com/tour-du-monde-en-80-jours-le-a125037930 Consulté le 16 décembre 2016.

51. http://lutinbazar.fr/escale-en-amerique-du-nord/ Consulté le 14 décembre 2016

52. http://mamaitressedecm1.fr/?p=1884 Consulté le 25 octobre 2016. 


\section{RÉSUMÉS}

Si les programmes de littérature pour l'école intègrent depuis 2002 la bande dessinée parmi les genres de textes à lire, le médium semble pourtant peu présent dans les classes. Le présent article propose un éclairage sur les pratiques de lecture de bandes dessinées à l'œuvre dans le cadre de l'enseignement de la littérature, à partir d'une étude de ressources proposées sur la blogosphère enseignante. Le statut des propositions publiées sur les blogs d'enseignants est d'abord interrogé, puis une analyse appuyée sur vingt-six séquences consacrées à la bande dessinée identifie des tendances en termes de corpus et d'activités prévues pour accompagner la lecture des bandes dessinées dans les classes.

Although graphic novels have been defined as a part of literature by the French literature curriculum for primary schools since 2002, they appear to be rarely read in literature classrooms. In order to better understand this situation, this article focuses on how graphic novels are actually taught when they enter the classroom. The survey is based on materials published on teachers' blogs : first, the methodological implications of such an investigation are taken into account, then 26 reading units are examined in order to analyse which graphic novels are read and what kind of reading tasks are most frequently designed.

INDEX

Mots-clés : bande dessinée, pratiques effectives, didactique de la littérature, blogs enseignants Keywords : graphic novels, teachers' practice, literature, education blogs

\section{AUTEUR}

HÉLÈNE RAUX

Université de Montpellier - LIRDEF 